\title{
Algunas consideraciones introductorias sobre la emisión de bonos high yield en el mercado de capitales internacional*
}

\author{
Rafael Lengua Peña** \\ Abogado por la Universidad de Lima. \\ Máster en Derecho (LL.M) "James Kent Scholar" por la Universidad de Columbia. \\ Abogado de Cleary, Gottlieb, Steen \& Hamilton. \\ Ex Miembro del Consejo Directivo de la Revista ADVOCATUS.
}

SUMARI0:

I. Introducción.

II. Ranking y subordinación:

1. Subordinación contractual;

2. Subordinación a deuda garantizada;

3. Subordinación estructural (structural subordination).

III. Análisis de los covenants y otros términos típicos en emisiones de bonos HY:

1. Incurrence covenants v. Maintenance covenants;

2. The box;

3. Clases de covenants:

3.1. Limitación a nuevos endeudamientos;

3.2. Restricción a nuevos pagos;

3.3.Limitación al otorgamiento de garantías sobre activos (negative pledge);

3.4. Limitación a la venta de activos;

3.5. Limitación a fusiones y ventas del negocio (merger covenant);

3.6. Prohibición de restricciones upstream;

3.7. Transacciones con afiliadas;

3.8. Limitación en la venta de participaciones minoritarias en las Restricted Subsidiaries.

4. Change of control output;

5. Limitaciones a la redención anticipada.

Dado que el idioma predominante para las emisiones de bonos HY en el mercado de capitales internacional es el inglés, en este artículo continuamente utilizaré anglicismos pues, en muchos casos, sería difícil o impráctico utilizar los términos en español.

** Las opiniones expresadas aquí son las opiniones del autor y no reflejan, necesariamente, las opiniones de Cleary Gottlieb Steen \& Hamilton. 


\section{INTRODUCCIÓN}

En el mercado de capitales internacional se denomina bonos high yield (HY) o de alto rendimiento a los bonos clasificados por debajo del "grado de inversión" por las tres mayores clasificadoras de riesgo - Moody's (> Ba1), Standard \& Poors (> BBB-) y Fitch (> BBB-) $-{ }^{1}$ que se emiten a largo plazo, a tasa de interés normalmente fija y sujetos a múltiples covenants -usualmente extensos y complejos.

Estos bonos se consideran de alto rendimiento, porque pagan un cupón más alto que los bonos grado de inversión para compensar el mayor riesgo asumido por los inversionistas -típicamente el yield spread entre bonos HY y bonos grado de inversión oscila entre 200 a 400 bps, aunque en épocas de crisis y alta volatilidad este spread puede ser mayor. ${ }^{2}$ A diferencia de los bonos grado de inversión, cuyo riesgo para los inversionistas está dado básicamente por el potencial incremento de las tasas de interés en el mercado, los bonos $\mathrm{HY}$-al igual que las acciones- son altamente sensibles a los riesgos específicos y sistémicos que afectan al emisor.

El alto rendimiento/riesgo puede ser consecuencia de aspectos vinculados a la estructura de los bonos y la finalidad para la cual se emiten, como en el caso de emisiones de deuda subordinada que sirven para complementar el financiamiento senior y mezzanine requerido por el sponsor de un leverage buyout ${ }^{3}$. También, serán de alto rendimiento si son emitidos por: (i) empresas intensivas en capital que tienen un alto grado de apalancamiento, (ii) start-ups que necesitan capital para expandir sus negocios y que carecen estabilidad financiera, utilidades, historial de ventas o activos suficientes para emitir bonos grado de inversión o conseguir financiamiento bancario, o (iii) empresas que desarrollan sus operaciones en países cuya deuda soberana no es clasificada como grado de inversión (conocido como "sovereing celling").

El boom de los bonos HY empezó a finales de la década de 1970 gracias a Michael Milken y el hoy fenecido banco de inversión "Drexel Burnham Lambert". Milken, hizo popular la idea que el alto riesgo de invertir en bonos HY -en esos tiempos conocidos como junk bonds- estaba más que compensado por el retorno esperado (i.e., el risk adjusted return por invertir en bonos HY era más alto que se conseguía por invertir en bonos grado de inversión), premisa fundamental para tomar una decisión de inversión. Desde la primera emisión suscrita por Bear Sterns en 1977, el mercado de bonos HY creció exponencialmente hasta que en 1989 sufrió una caída abrupta debido a reiterados incumplimientos producto de la sobreexposición a ciertas industrias, el gran tamaño de ciertas transacciones y la recesión estadounidense. La historia de los bonos HY en la década de 1980, finalmente,

1. La clasificación de riesgo se realiza, fundamentalmente, en función al nivel de apalancamiento del emisor, típicamente medido mediante ratios financieros como deuda total a EBITDA (utilidad operativa más cargos por depreciación y amortización), EBITDA a gastos financieros o EBITDA a fixed charges (servicio de deuda más dividendos fijos), riesgos específicos y sistémicos que afectan al emisor, la calidad de los covenants y algunos aspectos subjetivos tales como la calidad del management. Ver: Understanding Moody's Corporate Bond Ratings And Rating Process, en: http://www.moodys.com/ratings-process/Understanding-Moody-s-Corporate-Bond-Ratings-AndRating-Process/002005001.

2. Wells Fargo Advisors, A guide to investing in high-yield bonds: What you should know before you buy en: https://saf. wellsfargoadvisors.com/emx/dctm/Marketing/Marketing Materials/Fixed Income Bonds/E6578.pdf.

3. Por leverage buyout se entiende a las adquisiciones de empresas por firmas especializadas (hoy en día conocidas como private equity firms) utilizando una porción relativamente pequeña de capital y un alto grado de deuda de terceros. La deuda de terceros representa típicamente entre el 60 y 90 por ciento del precio de compra y está compuesta por deuda bancaria senior y garantizada y deuda junior en la forma de deuda mezzanine o bonos HY. Ver: KAPLAN, Steven N. y STROMBERG, Per. Leveraged Buyouts and Private Equity. En: Journal of Economic Perspectives, Vol. 22, No. 4 (2008). 
terminó con la quiebra de Drexel Burnham Lambert y el encarcelamiento de Milken por uso de información privilegiada. ${ }^{4}$

El mercado de bonos HY comenzó a recuperarse a medidos de la década de 1990 y es en esa época que comenzaron a ser denominados "bonos HY". La recuperación se dio gracias a la unión de la banca de inversión y comercial en los Estados Unidos producto de la derogación de la Glass Steagal Act, la aparición de numerosos hedge funds dedicados a invertir en bonos HY, el desarrollo de estructuras complejas que permitieron invertir en pools de bonos HY que diversificaban los riesgos específicos -conocidos como Collaterilized Debt Obligations o CDOs- y la globalización que amplió los mercados donde estos bonos podían ser ofertados y comercializados. ${ }^{5}$ Del 2003 al 2007, el mercado de bonos HY tuvo un alto crecimiento, cayendo fuertemente durante la reciente crisis financiera del 20082009. En el 2010 y 2011, el mercado de bonos HY ha venido mostrando algunos signos de recuperación. ${ }^{6}$

La base de inversionistas en bonos HY está representada por fondos mutuos especializados, sólo, en deuda HY o en bonos corporativos que buscan diversificar sus inversiones e incrementar el rendimiento del fondo (aproximadamente $35 \%$ ), fondos de pensiones que manejen recursos de pensionistas con un perfil de agresividad media (aproximadamente 25\%), empresas de seguros (aproximadamente 16\%), CDOs (aproximadamente 16\%) y hedge funds y otros inversionistas (el remanente).?

El objetivo de este artículo es explicar brevemente los aspectos más importantes de los bonos HY. Para ello, primero, describiremos los conceptos de ranking y subordinación y expondremos la manera cómo típicamente se mitiga el efecto de la subordinación en emisiones de bonos HY. Luego, pasaremos a analizar los covenants y demás términos usualmente contenidos en los indentures que regulan las emisiones de bonos HY.

\section{RANKING Y SUBORDINACIÓN}

El ranking de bonos HY y la potencial subordinación a otras deudas del emisor son factores muy importantes al analizar este tipo de emisiones. La subordinación puede tomar tres formas: (i) subordinación contractual; mediante la cual se acuerda expresamente subordinar los bonos a otras deudas del emisor, (ii) subordinación a deuda garantizada; según la cual los bonos son efectivamente subordinados a la deuda pari passu ${ }^{8}$ garantizada del emisor hasta por el monto del colateral, o (iii) subordinación estructural (structural subordination); según la cual los bonos se subordinan a la deuda de las subsidiarias del emisor.

\section{Subordinación contractual}

La subordinación contractual nace de un acuerdo entre las partes, mediante el cual se conviene

4. YAGO, Glenn. Junk Bonds. The Concise Encyclopedia of Economics. 2008. Library of Economics and Liberty. Recuperada: 28 de enero de 2012. En: http://www.econlib.org/library/Enc/JunkBonds.html.

5. Los mercados donde se emiten los bonos HY son el Euromercado, el cual proporciona financiamiento típicamente de tres a cinco años para emisores europeos y de mercados emergentes, y el mercado estadounidense que típicamente proporciona financiamiento de siete a diez años para emisores estadounidenses -en la última década se ha abierto a emisores de mercados emergentes.

6. Se estima que desde su aparición hasta el 2008, el mercado de bonos HY ha experimentado un crecimiento anual promedio (CAGAR) de más de 13\% y el valor de los bonos HY en circulación excede los US\$1,100 millones. Ver: REILLY, Frank K.; WRIGHT, David J. y GENTRY, James A. Historic Changes in the HY Bond Market, en http://business. illinois.edu/j-gentry/workshop/exhibit-14.pdf.

7. Datos al 2004, en: Standard \& Poor's LCD High-Yield Bond Marker Primer. En: https://www.lcdcomps.com/d/pdf/ hyprimer.pdf.

8. El término pari passu significa "igual" y es usado comúnmente para referirse a la intención de las partes que la deuda este en el mismo nivel, sin preferencia o prioridad. Para una brillante exposición del tema. Ver, BUCHHEIT, Lee C. y PAM, Jeremiah S. The Pari Passu Clause in Sovereign Debt Instruments, 53 EMORY L.J. 869, 895 (2004). 
expresamente que la deuda senior ${ }^{9}$ tendrá preferencia de pago frente a la deuda subordinada, si el deudor es sometido a proceso de insolvencia o liquidación (liquidation subordination).$^{10} \mathrm{En}$ la medida que este tipo de subordinación nace en virtud de un contrato y las leyes de insolvencia son generalmente de orden público, resulta importante confirmar que será exigible en un escenario de insolvencia según las leyes aplicables al deudor. ${ }^{11}$

Además de la prioridad en el pago en caso de insolvencia o liquidación, los pactos de subordinación suelen contener restricciones de pagos (standstill provisions) que conceden a los titulares de deuda senior, derechos a la suspensión del pago del principal e intereses correspondientes a la deuda subordinada (payment subordination). ${ }^{12}$ Primero, los pactos de subordinación establecen que si la deuda senior es acelerada, el emisor automáticamente deberá suspender el pago del principal e intereses de la deuda subordinada. Segundo, se dispone que si el deudor incumple el pago de interés o principal o algún covenant correspondiente a la deuda senior -y ésta no es acelerada- los titulares de deuda senior tendrán derecho a que el deudor suspenda el pago de interés y principal de de la deuda subordinada por un periodo de 179 días para permitir una salida negociada al problema -conocido como "blocking right".

\section{Subordinación a deuda garantizada}

Salvo en el caso de asset-backed financings, donde el financiamiento es garantizado por activos específicos del deudor -por ejemplo, cuentas por cobrar e inventarios en un borrowing base facility o equipos adquiridos en un equipment financing- es común que los financiamientos bancarios a emisores de bonos HY estén garantizados por todos sus activos fijos o al menos sus activos fijos más importantes.

En un escenario de insolvencia, los acreedores garantizados tendrán prioridad frente a los titulares de bonos HY hasta por el $100 \%$ del producto de la venta del colateral $y$, además, tendrán el mismo derecho que los titulares de bonos HY a la distribución proporcional de los activos remanentes en caso el colateral no resulte suficiente. Para los titulares de bonos HY -y demás acreedores no garantizados- esto tiene un efecto de subordinación, pues proporciona a los acreedores garantizados un fondo preferente de repago sobre activos del deudor en un escenario de insolvencia. ${ }^{13}$

9. Si bien puede variar, la deuda senior es normalmente definida de manera amplia e incluye deuda con bancos y la deuda existente o subordinada que no esté expresamente subordinada a los bonos y a otra deuda del emisor.

10. Los titulares de deuda senior son designados expresamente en el indenture como third-party beneficiaries de la subordinación contractual. Bajo el common law, el concepto de third-party beneficiary es una excepción al principio de privity of contracts y permite que un tercero pueda demandar al promitente, incluso si la promesa fue realizada a favor del destinatario y no del third-party beneficiary, siempre que la promesa haya estado encaminada a beneficiar al third-party beneficiary.

11. Por ejemplo, en los Estados Unidos de América la Sección 510(a) del Bankruptcy Code expresamente reconoce los pactos de subordinación en procesos de insolvencia. Para un interesante estudio sobre la exigibilidad de pactos de subordinación en países de Europa del Este. Ver, THEISS, Wolf, Restructuring Loans and Enforcement of Security (2010).

12. Estos pactos, sin embargo, rara vez estarán presentes en bonos HY emitidos por oferta pública en los Estados Unidos o emitidos por oferta privada en el mercado de capitales internacional con registration rights en los Estados Unidos. La razón es que en este tipo de emisiones el indenture tendrá que ser calificado bajo el Trust Indenture Act de 1939 y existe la visión mayoritaria que los standstill provisions atentan contra la Sección 316(b) del Trust Indenture Act de 1939 la cual otorga a los bonistas el derecho de exigir el pago de principal e intereses ante un incumplimiento. Ver: WILKINSON, Andrew y LUCAS, Andrew. High yield bond issues-Raising the stakes, PLC December 2003, p.20.

13. Este tipo de subordinación está más enfocada en cómo se distribuye la masa concursal ante una eventual liquidación del deudor (liquidation subordination) que en restricciones a pagos a otros acreedores antes de la liquidación (payment subordination). 


\section{Subordinación estructural (structural subordination)}

La subordinación estructural (structural subordination), se presenta cuando el emisor tiene subsidiarias operativas con flujos de caja, activos y pasivos que son parte importante de la empresa consolidada. En este caso, salvo que las subsidiarias garanticen la deuda, los acreedores del emisor -garantizados, senior y junior- estarán estructuralmente subordinados a los acreedores de las subsidiarias. Esto es así, porque en un escenario de insolvencia los acreedores de las subsidiarias tendrán preferencia en la distribución de la masa concursal, esto es, tendrán derecho a ser pagados antes que se puedan distribuir activos al emisor $y$, por ende, a sus acreedores.

El diagrama que sigue a continuación, explica el efecto de la subordinación estructural típicamente presente cuando un emisor de bonos $\mathrm{HY}$ tiene una subsidiaria operativa con accionistas minoritarios. Asumiendo que, en un escenario de insolvencia los activos de la subsidiaria son vendidos por $\$ 100$, primero se cobrarían el acreedor senior $(\$ 30)$, el acreedor legal $(\$ 20)$ y el acreedor subordinado $(\$ 20)$ y luego el remanente $(\$ 30)$ sería distribuido a pro rata entre el accionista minoritario (\$3) y el emisor (\$27), siendo este último monto la única cantidad de dinero disponible para los titulares de bonos HY.
El objetivo al estructurar una emisión de bonos HY siempre será mitigar los efectos de la subordinación estructural, pues ésta aumenta el riesgo asignado a los bonos por las clasificadoras de riesgo y los inversionistas lo que no sólo aumenta el rendimiento que los inversionistas van a exigir sino que, además, puede afectar el marketing y distribución por parte de los estructuradores.

Esta tarea puede ser particularmente desafiante cuando la estructura corporativa del emisor es compleja y tiene subsidiarias en diferentes jurisdicciones.

Básicamente, hay tres soluciones para mitigar los efectos de la subordinación estructural: (i) conseguir garantías upstream y pari passu de las subsidiarias, (ii) limitar el endeudamiento de las subsidiarias, o (iii) revelar a los inversionistas la existencia de la subordinación estructural y asumir los efectos de la menor clasificación de riesgo y el mayor costo de la deuda.

El otorgamiento de garantías upstream y pari passu por parte de las subsidiarias convierte a titulares de bonos HY en acreedores de las subsidiarias del emisor. Si bien, el otorgamiento de garantías upstream es la mejor manera de afrontar la subordinación estructural, puede generar algunas complicaciones que deben analizarse cuidadosamente en cada emisión.

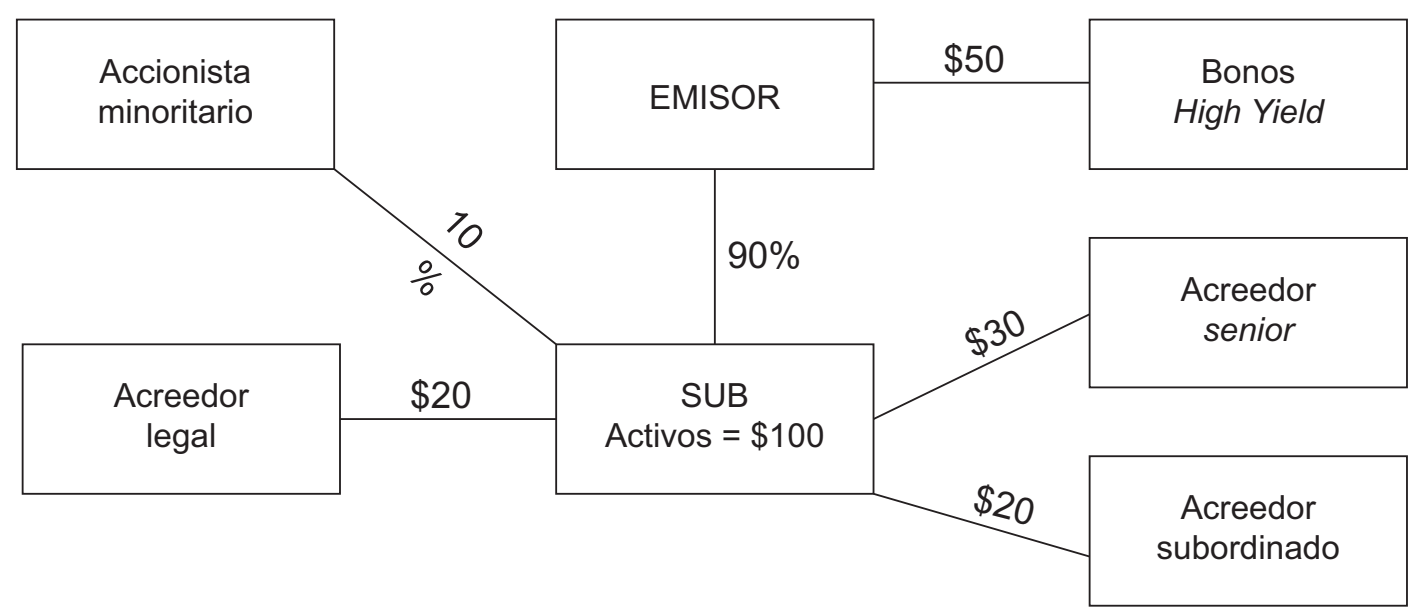


Primero, en ciertas jurisdicciones -como, por ejemplo, los Estados Unidos- las garantías otorgadas por subsidiarias pueden ser declaradas inválidas ante una eventual insolvencia si constituyen fraudulent conveyance. ${ }^{14}$ Segundo, las garantías upstream son problemáticas cuando la subsidiaria tiene accionistas minoritarios no afiliados al emisor, porque los accionistas minoritarios asumirán el riesgo financiero de la matriz sin necesariamente obtener beneficios equivalentes. Esto puede generar la violación de derechos de los accionistas minoritarios o el incumplimiento de los deberes fiduciarios del emisor, en su calidad de accionista controlador, y de los directores de la subsidiaria, lo cual debe ser analizado cuidadosamente. Tercero, una subsidiaria que tenga acreedores contractuales o accionistas minoritarios que cuenten con algún acuerdo previamente negociado y suscrito ${ }^{15}$, probablemente, estará obligada a no otorgar garantías bajo algún covenant existente.

Otra alternativa para mitigar la subordinación estructural, especialmente cuando existen restricciones para otorgar garantías upstream, es limitar el endeudamiento de las subsidiarias. Si bien, esta alternativa también resulta satisfactoria para las clasificadoras de riesgo y los inversionistas, es mucho menos efectiva que las garantías upstream y, por ende, rara vez resulta ser la primera opción de los estructuradores. En particular, por razones prácticas y legales, no se puede limitar el endeudamiento frente a trade creditors - de lo contrario la subsidiaria no podría financiar el pago de activos o servicios sin el consentimiento de los bonistas- o frente a acreedores legales -que pueden representar parte importante de los pasivos de la subsidiaria dependiendo de la industria del emisor. ${ }^{16}$

Finalmente, es posible lidiar con la subordinación estructural simplemente revelándola a los inversionistas para que estos determinen el nivel de riesgo y lo trasladen al emisor aumentando el rendimiento esperado (i.e., la tasa de interés).

\section{ANÁLISIS DE LOS COVENANTS Y OTROS TÉRMINOS TÍPICOS EN EMISIONES DE BONOS HY}

En todo contrato de deuda, particularmente los relativos a deuda $\mathrm{HY}$, los covenants juegan un papel muy importante, pues mitigan el riesgo de moral hazard producto del oportunismo post-contractual de los deudores. Este moral hazard se genera, porque económicamente existe una relación de agencia ${ }^{17}$ entre deudor y los acreedores en la cual, el deudor tiene incentivos a satisfacer sus propios intereses antes que los intereses de los acreedores, sea desviando recursos fuera del alcance de los acreedores, subordinando a los acreedores a nuevas deudas o adoptando decisiones altamente riesgosas o que puedan afectar los intereses de los bonistas.

En empresas donde el control está concentrado en un grupo de accionistas, esta relación también puede ser vista económicamente como una en la cual los accionistas tienen una opción de compra que les permite recomprar los acti-

14. Bajo la doctrina de fraudulent conveyance, el juez puede invalidar una garantía upstream si considera que la subsidiaria no ha recibido un valor razonablemente equivalente por otorgar las garantías y estas han sido otorgadas en un periodo cercano a la insolvencia, entre otros requisitos. Por esta razón, salvo que esto no sea un riesgo, típicamente las garantías upstream limitan el monto de las garantías que cada subsidiaria puede otorgar a aquel monto que no causaría su invalidación bajo la doctrina del fraudulent conveyance (esto se conoce como "savings clause").

15. Esto es bastante común en el caso de joint ventures o cuando se incorpora un accionista minoritario (ej. fondos de prívate equity o accionistas estratégicos) luego de un proceso de venta privada.

16. Estos acreedores serían pari passu con los bonistas si la subsidiaria otorga una garantía upstream, pero senior si no hubiera garantía. De la misma manera, los accionistas minoritarios de la subsidiaria serían junior a los bonistas si hubiera una garantía upstream y pari passu si no la hubiera.

17. Para una mayor comprensión del concepto, ver: JENSEN, Michael J. y MECKLING, William H., Theory of the firm: managerial behavior, agency costs, and ownership structure, 3 J. Fin. Econ. 305 (1976). 
vos de la empresa. ${ }^{18}$ Como en el caso de cualquier opción de compra, los accionistas sólo la ejercitarán si el valor de los activos subyacentes (i.e., el valor de la empresa) es mayor al precio por repagar la deuda (strike price). En la medida que incrementos en la varianza (i.e., posibilidad de variación de precios) sobre el valor futuro de los activos subyacentes incrementan el valor de la opción, los accionistas tendrán grandes incentivos para tomar decisiones riesgosas que afectan los intereses de los acreedores. Los Covenants, vistos desde esta perspectiva, buscan reducir la varianza del valor de los activos subyacentes limitando el comportamiento estratégico de los deudores.

Toda vez que, el riesgo de moral hazard es mayor en el caso de emisores clasificados por debajo del grado de inversión, los covenants aplicables a bonos HY son bastante más extensos y complejos que los aplicables a una emisión de bonos grado de inversión y, además, usan un lenguaje altamente técnico por estar vinculados fundamentalmente a medidas contables y financieras. Por ello, para quienes no están familiarizados con ellos -hecho que por lo general afecta a emisores que por primera vez emiten bonos HY en el mercado de capitales internacional- no es extraño que sean complicados de entender.

Para entender estos covenants, es necesario comprender que su objetivo primordial es limitar acciones del emisor que puedan afectar su habilidad de repagar los bonos y el servicio de deuda sin que ello impida el desarrollo diario de sus operaciones y plan de negocios. ${ }^{19}$ Para esto, primero, es necesario analizar las políticas contables del emisor, sus proyecciones, plan de negocios y estructura legal para entender cuáles son los activos y operaciones materiales del emisor y riesgos inherentes al mismo y el grado de flexibilidad que éste requiere para manejar sus negocios. Es importante, también entender que existe una diferencia importante entre incurrence covenants -típicamente contenidos en los indentures de bonos HY- y maintenance covenants -típicamente contenidos en contratos de crédito bancario- $y$ delimitar lo que en el mercado se conoce como "the box", esto es, el universo de sociedades a las cuales se le aplican estos covenants. Finalmente, debe tenerse en consideración que los términos contables y financieros utilizados en el indenture muchas veces son distintos que los utilizados por el emisor para reportar sus resultados contables y financieros por estar limitados únicamente a medidas contables y financieras que se generen dentro del box.

\section{Incurrence covenants v. maintenance covenants}

Los covenants son deberes contractuales, mediante los cuales se establecen ciertos estándares de conducta que deben ser cumplidos por el deudor durante la vigencia del contrato de deuda. ${ }^{20}$ Cuando hablamos de un incurrence covenant estamos haciendo referencia a un deber contractual que limita la libertad del emisor de realizar ciertas acciones -como, por ejemplo, tomar deuda, otorgar garantías a terceros, pagar dividendos- si no se satisface un test financiero o si no califica dentro de alguna de las excep-

18. En efecto, el acuerdo entre accionistas y acreedores puede ser visto como la venta a los acreedores de una empresa sin endeudamiento a cambio de: (i) los fondos recibidos al tomar la deuda, (ii) un contrato de gerencia, y (iii) un call option que les permite recomprar los activos de la empresa al repagar la deuda a su valor nominal más intereses. En la fecha de repago, si los activos de la empresa valen más que el precio de repago de la deuda, los accionistas ejercitarán su opción y recomprarán los activos de la empresa pagando la deuda. Si, por el contrario, los activos de la empresa valen menos que el precio de repago de la deuda, lo accionistas no ejercitarán su opción y simplemente incumplirán la deuda. Ver: SCHOLES, Myron. Options - Puts and Calls, en: Encyclopedia of Investments (BLUME, Marshall y FRIEDMAN, Jack. eds.1982), pp. 559-578. GILSON, Ronald J. y BLACK, Bernard. The Law and Finance of Corporate Acquisitions, Supplement, Capítulo 7 (1992).

19. Ver: NOWAK, Gerald T. The Gift that Keeps on Giving: Negotiating the High Yield Indenture, Venture Capital Review, No. 15 (2005).

20. PAGLIA, John K. An Overview of Covenants in Large Bank Loans. RMA Journal (Mar. 2002). BNET. Web. 7 Mar. 2010. 
ciones previamente establecidas (conocidas como "carveouts"). Por el contrario, cuando nos referimos a un maintenance covenant aludimos a un deber contractual que debe ser cumplido por el emisor de manera constante -aunque usualmente medidos al final de ciertos periodos de tiempo, por ejemplo, trimestralmente- durante el plazo de la deuda.

Aunque, la diferencia puede parecer sutil o incluso inexistente, entenderla es muy importante si queremos comprender las consecuencias de estos covenants para el emisor. En la prácti$\mathrm{ca}$, los maintenance covenants son mucho más estrictos que los incurrence covenants y pueden tener consecuencias indeseadas para el emisor en una emisión de bonos HY. ${ }^{21}$ Por ejemplo, supongamos que ambos covenants establecen un ratio de "pro forma" EBITDA a fixed charges ("ratio de cobertura") máximo de 3.0x. Un típico incurrence covenant en un bono HY prohibiría al emisor tomar deuda salvo que el ratio de cobertura sea de por lo menos 3.0x, luego de considerar la nueva deuda. Un maintenance covenant, en un contrato de crédito exigiría al emisor mantener un ratio de cobertura de por lo menos 3.0x, durante cada trimestre. Esto quiere decir que si el emisor asumió un incurrence covenant y su ratio de cobertura cae debajo de 3.0x, la consecuencia sería únicamente que el emisor no puede tomar más deuda que no califique dentro de alguno de los carveouts hasta que se satisfaga el ratio. En ningún caso, el emisor estaría en default por no cumplir con el ratio. Por el contrario, bajo un maintenance covenant, si en un determinado trimestre el ratio de cobertura cae por debajo de 3.0x, el emisor sí estaría en default.

El uso de maintenance covenants hace sentido, básicamente, en financiamientos bancarios porque sirven como sistema de alerta previa a un default, permitiendo que los acreedores y el deudor negocien la dispensa anticipada del covenant a cambio de una mayor tasa de interés u otra forma de compensación. Es muy riesgoso incluir un maintenance covenants en un bono HY, porque ante un incumplimiento cualquier modificación o dispensa requeriría la aprobación de por lo menos la mayoría de los titulares de bonos lo que puede tomar tiempo y ser complicado de conseguir si se presentan holdouts o problemas de acción colectiva.

\section{The box}

El segundo concepto que debe entenderse cuando se analizan bonos HY es el de "the box", esto es, el universo de sociedades afiliadas al emisor que servirán para el repago de los bonos HY y a las cuales se le aplican los diversos covenants. Se considera que están dentro del box: el emisor y todas sus subsidiarias, salvo aquellas que el emisor y los bancos acuerden dejar fuera para, por ejemplo, permitir el desarrollo de startups que pueden generar pérdidas iniciales o joint ventures estratégicos. Las subsidiarias que están dentro del box se denominan "Restricted Subsidiaries" porque están restringidas por los covenants, mientras que las que quedan fuera se conocen como "Unrestricted Subsidiaries".

El objetivo de los estructuradores será siempre minimizar (i) la cantidad de activos fuera del box, (ii) el monto de acreencias senior sobre los activos que están dentro del box, y (iii) el monto total de acreencias que tengan recurso sobre los activos que están dentro del box. Mientras tanto, el objetivo del emisor será maximizar su flexibilidad para perseguir sus objetivos de negocios y desarrollar proyectos futuros.

El concepto de "the box" se basa en las siguientes asunciones que se regulan en el indenture: (i) los covenants y cualquier pedido de garantías upstream típicamente sólo se aplican al emisor y las "Restricted Subsidiaries"; (ii) al calcular la deuda consolidada, ingresos y fixed charges para efectos de los test financieros usados en los covenants, sólo se incluye la deuda, ingresos y fixed

21. Nomura International PLC. Global Guide to Corporate Bankruptcy: A comprehensive guide to corporate bankruptcy and a survey of global corporate bankruptcy regimes. http://www.scribd.com/doc/78673927/10/The-covenantbreach-process. 
charges del emisor y las "Restricted Subsidiaries"; y (iii) todas las inversiones hechas por el emisor y las "Restricted Subsidiaries" (incluyendo inversiones en Unrestricted Subsidiaries), excepto por aquellas hechas en las "Restricted Subsidiaries", sólo pueden ser realizadas en cumplimiento del restricted payments covenant. ${ }^{22}$ el siguiente diagrama ayuda a graficar esto:

\section{Clases de covenants}

A diferencia de los bonos grado de inversión donde las actividades del emisor, únicamente, se limitan mediante prohibiciones al otorgamiento de garantías (negative pledge) o operaciones de venta y leaseback, ${ }^{23}$ los indentures de bonos HY contienen una gran cantidad de covenants.

Sujeto a varias excepciones y calificaciones que serán explicadas a continuación, estos covenants fundamentalmente dicen lo siguiente: (i) no debe aumentarse el apalancamiento, porque los inversionistas invirtieron en los bonos basados en el endeudamiento al momento de la emisión y aquel proyectado en el plan de negocios; (ii) no deben distribuirse o invertirse recursos fuera del propio negocio, si se hace es mejor usar los fondos para repagar la deuda; (iii) no deben venderse activos salvo que el precio sea utilizado para reinvertir en el negocio, reducir el endeudamiento o repagar los bonos HY; (iv) no deben otorgarse garantías sobre activos, éstos son más valiosos para los bonistas si no están gravados; (v) no deben realizarse transacciones fuera de mercado con afiliadas, éstas sólo transfieren valor fuera del negocio y los bonistas reciben nada a cambio; (vi) las subsidiarias que no garanticen los bonos no deben endeudarse o garantizar deudas, esto subordinaría estructuralmente a los bonistas $\mathrm{HY}$ frente a los nuevos acreedores; (vii) no deben imponerse restricciones a las subsidiarias para repartir dividendos o realizar pagos a accionistas que dificulten el repago de los bonos; y (viii) no deben venderse acciones en las subsidiarias, pues los titulares de bonos HY quieren tener derecho al íntegro de las distribuciones realizadas por estas subsidiarias. ${ }^{24}$

\subsection{Limitación a nuevos endeudamientos}

Mediante este covenant, el emisor se compromete a no incurrir, directamente o a través de alguna de las Restricted Subsidiaries, en nuevos endeudamientos (incluyendo garantías a deudas de terceros), salvo que ello esté expresamente permitido en el indenture. Los indentures de bonos HY típicamente permiten que el emisor o las Restricted Subsidiares que garanticen los bonos $\mathrm{HY}$ incurran en nuevos endeudamientos

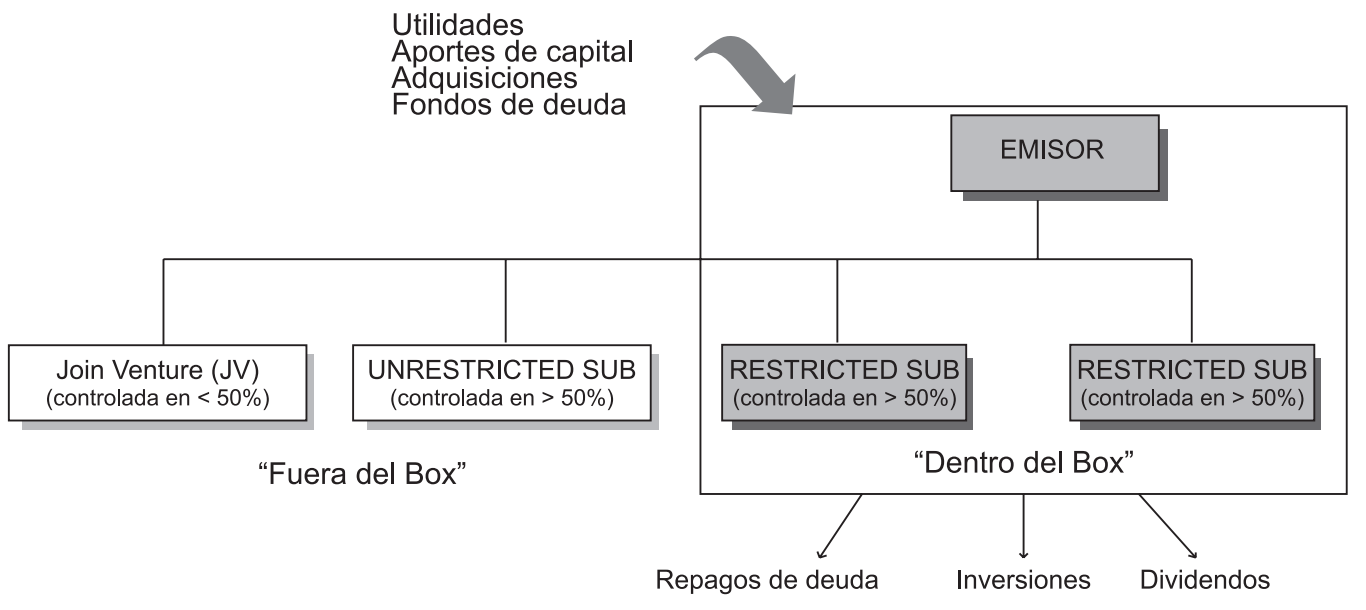

22. WEBB, David. Documentation for High Yield Debt, Euromoney Books (2011), p.15.

23. Ver, The Credit Roundtable, Improving Covenant Protections in the Investment Grade Bond Market (2007) en: http:// www.iimemberships.com/dl/creditroundtable/Covenant\%20White\%20Paper\%20revised\%207-2-08.pdf.

24. NOWAK, Gerald T. Supra nota 18, p. 2. 
siempre que: (i) el ratio de cobertura (o el ratio de deuda total a EBITDA, de ser el caso $)^{25}$ sea superior al ratio acordado entre el emisor y los estructuradores ("leverage test") ${ }^{26}$; o (ii) la nueva deuda califique dentro de alguna de las excepciones al covenant (conocidas como permitted debt carveouts ${ }^{27}$ ).

Este covenant busca limitar el aumento del riesgo de incumplimiento del emisor, mediante el incremento de la deuda total o su servicio de deuda relativo a EBITDA y mitigar el riesgo de subordinación estructural que puede producirse si el emisor incrementa su endeudamiento a través de alguna de las Restricted Subsidiaries que no garanticen los bonos. En la medida que este covenant no debe limitar la operación ordinaria de los negocios del emisor, típicamente se permite que el emisor y las subsidiarias que garanticen los bonos incurran en nuevos endeudamientos si se cumple el leverage test o si se califica dentro de alguna de las excepciones previamente acordadas. Por esta razón, la correcta determinación ex ante del leverage test y de los carveouts es muy importante y debe ser evaluada conjuntamente entre el emisor y los estructuradores en función de las proyecciones del negocio (en particular, la proyección de EBITDA) y sus necesidades de financiamiento.

\subsection{Restricción de pagos}

Según este covenant el emisor y las Restricted Subsidiaries se comprometen a no pagar dividendos en dinero, realizar distribuciones a accionistas (salvo distribución de acciones) o recomprar acciones ("dividend stopper"), pre-pagar o recomprar deuda subordinada a los bonos o realizar inversiones fuera del box (definidos como "Restricted Payments") salvo que: (i) se satisfaga el siguiente test tripartito: (a) el pago no genere un incumplimiento o evento de incumplimiento; (b) se cumpla el leverage test, y (c) la suma de Restricted Payments realizados desde la emisión de los bonos HY (incluyendo el que se piensa efectuar) no exceda la suma acumulada de (y) 50\% del resultado neto (consolidado) del emisor (o 100\% si el resultado neto es negativo) y (z) 100\% de los fondos recibidos producto de aportes de capital (el producto de esta operación aritmética es conocido como "earnings/equity basket"); ${ }^{28} \mathrm{o}$ (ii) los pagos estén expresamente incluidos dentro de la lista de inversiones permitidas ("Permitted Investments"29) o exceptuados de la aplicación de este covenant. ${ }^{30}$

25. Tal vez los dos ratios más importantes que los inversionistas analizan al evaluar y valorizar los bonos HY son el ratio de cobertura (EBITDA a fixed charges) y el ratio deuda total a EBITDA, usualmente utilizado en la industria de telecomunicaciones o prensa.

26. Usualmente el ratio de cobertura será 2.0 a 1.0, aunque algunas veces es mayor o se acuerdan step-up que incrementan el ratio con el pasar de los años. Ver: NOWAK Gerald T. , supra nota 19, p. 5.

27. Los típicos carveouts en un indenture de bonos HY son: (i) deuda producto de un reopening de los bonos hasta por un monto determinado; (ii) deuda bancaria hasta por un monto determinado; (iii) deuda contraída bajo derivados de cobertura; (iv) deuda subordinada entre el emisor y las Restricted Subsidiaries; ( $v$ ) deuda incurrida dentro del curso ordinario de los negocios; (vi) deuda incurrida para refinanciar los bonos HY o deuda preexistente; y (vii) deuda incurrida hasta por un cierto monto para adquirir activos o celebrar arrendamientos financieros o para usos generales.

28. En algunos casos, el emisor puede requerir un basket general para realizar Restricted Payments el cual puede ser agregado al earnings equity basket o ser utilizado incluso si no se cumple el leverage test o el earnings/equity basket es negativo. WEBB, supra nota 22, pp. 60-61.

29. Dentro de la lista de Permitted Investments típicamente se encuentran (i) las inversiones dentro del box (i.e., inversiones en Restricted Subsidiaries o en el emisor), (ii) inversiones dentro del curso ordinario de los negocios, como por ejemplo, inversiones del área de tesorería en equivalentes de efectivo, (iii) inversiones producto de pagos en especie recibidos por vender activos en cumplimiento con el covenant respectivo, (iv) inversiones por una única vez hasta por un monto determinado.

30. Tal es el caso de pagos realizados (i) producto de intercambios de acciones (siempre que no sean acciones que deben ser recompradas o redimidas en el futuro, i.e., "Disqualified Stock"), o (ii) para refinanciar deuda subordinada mediante la emisión de acciones o deuda subordinada junior a los bonos HY (siempre que no tenga un duration menor a la deuda subordinada que está siendo refinanciada). 
En general, este covenant busca limitar la transferencia de recursos fuera del box. La restricción de pagos a accionistas - dividendos, distribuciones o recompra de acciones- y acreedores subordinados busca restringir transferencias de recursos a titulares de instrumentos de capital o deuda subordinados a los bonos HY que no puedan ser financiados con resultados acumulados o aportes de capital en un escenario en que el emisor no satisfaga el leverage test. La restricción aplicable a inversiones en Unrestricted Subsidiaries o inversiones minoritarias en entidades no relacionadas al emisor busca limitar la transferencia de recursos a empresas fuera del box, porque éstas no están sujetas a los covenants $y$, además, porque los titulares de bonos HY estarán estructuralmente subordinados lo que les confiere acceso limitado a los flujos de caja que resulten de esas inversiones.

\subsection{Limitación al otorgamiento de garantías sobre activos (negative pledge)}

Mediante este covenant, el emisor y las Restricted Subsidiaries se obligan a no contraer deuda garantizada con sus activos si no se garantizan los bonos HY de la misma manera y sobre los mismos activos o estás garantías se encuentran listadas dentro de las excepciones a este covenant conocidas como "Permitted Liens"31. Su finalidad, es limitar el monto de nueva deuda senior a los bonos HY para que, en un escenario de insolvencia, los activos del emisor estén disponibles para pagar a los bonistas pro rata con los otros acreedores.

\subsection{Limitación a la venta de activos}

Según este covenant, salvo por algunas excepciones, el emisor y las Restricted Subidiaries se obligan a no vender activos, salvo que la venta se lleve a cabo a valor de mercado y, por lo menos, entre el 75\%-85\% de la contraprestación sea en efectivo y utilizada dentro de 180-365 días para (i) capex dentro del box o (ii) repagar deuda garantizada o senior a los bonos HY (incluyendo deuda estructuralmente senior). $\mathrm{Si}$ los fondos no son utilizados dentro del plazo establecido conforme se indicó anteriormente, el emisor deberá realizar una oferta de recompra de los bonos HY a la par, salvo que los activos vendidos no superen un monto establecido en función del tamaño de la oferta.

La racionalidad de este covenant es mitigar la posible reducción de flujos de caja producto de la venta de activos. Por ello, sólo se podrán vender activos si son reemplazados con nuevos activos, se reduce el endeudamiento ( $y$, por ende, el servicio de deuda) mediante el repago de deuda garantizada o senior, o si se otorga la oportunidad a los bonistas de salirse de su inversión.

Es importante notar que, si bien este covenant no debería afectar la operación ordinaria de los negocios del emisor y las Restricted Subsidiaries, puede limitar su habilidad de realizar joint ventures con terceros mediante aportes de activos, financiar la venta de activos, o vender activos de la compañía sujetos a un earn out donde parte de la contraprestación deberá ser pagada en el futuro dependiendo de los resultados obtenidos. ${ }^{32}$

\subsection{Limitación a fusiones y ventas del negocio (merger covenant)}

Este covenant, limita operaciones de fusión o la venta de todos o sustancialmente todos ${ }^{33}$ los activos del emisor en los siguientes tres supues-

31. Incluyen cargas y gravámenes impuestos: (i) por ley, requerimientos ordinarios de entidades gubernamentales o prácticas de mercados en el curso ordinario de los negocios (por ejemplo, gravámenes garantizando cartas de crédito para exportación), (ii) por el vendedor sobre un activo vendido al crédito, (iii) sobre inventarios o derechos a recibir inventario, (iv) para refinanciar deuda existente y, (v) en relación con deuda incurrida en cumplimiento con el covenant sobre limitación de endeudamientos.

32. WEBB, supra nota 22, p. 65.

33. La determinación sobre qué constituyen substancialmente todos los activos del emisor no ha sido definida con precisión por la jurisprudencia de las cortes de Nueva York, a cuya ley usualmente se someten los indentures y 
tos: (i) el emisor no es la empresa que sobrevive la fusión o ésta no asume la deuda, (ii) producto de la fusión o venta, la entidad sobreviviente o el adquirente no satisfacen el levarage test, o (iii) la empresa fusionada o el adquirente tienen patrimonio negativo.

La racionalidad de este covenant es impedir que mediante fusiones o ventas a empresas apalancadas, se incremente indirectamente el endeudamiento, se desvíen recursos fuera del box, o se reduzca el patrimonio neto de las empresas dentro del box.

\subsection{Prohibición de restricciones upstream}

A través de este covenant, se exige que el emisor cause que las Restricted Subsidiaries no acuerden con terceros un covenant similar al covenant de restricción de pagos descrito anteriormente que limite su habilidad de realizar transferencias upstream al emisor u otras Restricted Subsidiaries.

Los inversionistas evalúan al emisor en función de su situación consolidada, la cual asume que el emisor tiene acceso a los recursos de las Restricted Subsidiaries. Si el emisor se comprometiera a un covenant que restrinja pagos upstream, limitaría su a fondos provenientes de las Restricted Subsidiaries y, por ende, podría afectar su habilidad pagar su deuda o financiar sus negocios. Por ello, la racionalidad de este covenant es preservar el acceso del emisor a los recursos dentro del box que vengan de las Restricted Subsidiaries.

\subsection{Transacciones con afiliadas}

Mediante este covenant se obliga al emisor, directamente y a por cuenta de sus Restricted Subsidiaries, a realizar transacciones con empresas afiliadas fuera del box en términos que no sean menos favorables que aquellos que serían obtenidos en una transacción comparable y en arms-length realizada con una persona que no sea afiliada al emisor. Salvo por transacciones en el curso ordinario de los negocios, esta determinación se realiza en dos niveles.

En un primer nivel, hasta por un monto a ser determinado en función del tamaño del emisor, la determinación es hecha por el directorio - compuesto por una mayoría de directores desinteresados. En un segundo nivel, si la transacción supera el monto indicado anteriormente, esta determinación deberá ser realizada por un auditor independiente, tasador o banco de inversión.

La finalidad de este covenant es limitar las transferencias de recursos hacia empresas afiliadas fuera del box que no estén capturadas dentro del covenant de restricción de pagos pues es una manera de transferir recursos fuera del alcance de los titulares de bonos HY.

\subsection{Limitación en la venta de participaciones minoritarias en las Restricted Subsidiaries}

Por medio de este covenant se impide que terceros adquieran o sean titulares participaciones minoritarias en el capital social de las Restricted Subsidiaries, salvo en ciertas transacciones con miembros del directorio, adquisiciones de $100 \%$ del capital social de las Restricted Subsidiaries, acciones recibidas como dividendos en especie, o acciones transferidas producto de una emisión de acciones hasta por un porcentaje minoritario previamente determinado.

La racionalidad de este covenant es proteger a los titulares de bonos HY contra la subordinación estructural y asegurar que los fondos

contratos financieros que contienen este covenant. En: Sharon Steel Corp. V. the Chase Manhattan Bank et. al. 691 F.2d 1039 (2d. Cir. 1982), uno de los casos más relevantes sobre la materia, la corte de apelaciones de Nueva York analizó tanto factores cuantitativos, tales como el porcentaje que representan los activos transferidos frente a los activos totales del emisor, su contribución al flujo de caja del emisor, como cualitativos, tales como el riesgo que la venta afecte la operación normal de los negocios de emisor. 
provenientes de las Restricted Subsidiaries no sean compartidos con terceros.

\section{Change of control put}

El change of control put es una disposición que confiere a los titulares de bonos $\mathrm{HY}$, el derecho a exigir que el emisor ofrezca recomprar todo o parte de los bonos HY a un precio igual a $101 \%$ de su valor nominal más intereses devengados si ocurre un cambio sustancial en la estructura de propiedad o administración del emisor o la matriz del emisor. ${ }^{34} \mathrm{Si}$ el emisor incumple con realizar esta oferta de recompra, ello será considerado como un evento de incumplimiento.

El propósito de esta disposición es permitir que los bonistas evalúen nuevamente su decisión de invertir en los bonos si el emisor o su matriz son adquiridos por un tercero. ${ }^{35}$ En la medida que los bonos $\mathrm{HY}$ son sensibles a riesgos específicos del emisor, cambios en el management del emisor o su accionista controlador pueden tener efectos negativos para los inversionistas. Además, conjuntamente con el merger covenant, esta disposición otorga protección a los bonistas frente a reestructuraciones corporativas que puedan afectar sus intereses y adquisiciones hostiles.

\section{Limitaciones a la redención anticipada}

Como todo instrumento de renta fija, los bonos están sujetos a las variaciones de las tasas de interés en el mercado. El emisor, por tanto, tendrá incentivos para redimir anticipadamente los bonos $\mathrm{HY}$ si los intereses que paga son mayores que los que pagaría en una nueva emisión de bonos o financiamiento bancario. Esta redención, por el contrario, será perjudicial para los inversionistas porque éstos perderán los flujos de caja esperados y asumirán el riesgo de no poder reinvertir el capital a una tasa de retorno equivalente. Es por esto que, típicamente, en una emisión de bonos HY se prohíbe que el emisor, los redima durante la primera mitad del plazo de duración.

Pese a esta limitación, usualmente se permiten redenciones anticipadas producto de lo que se conoce como "equity clawbacks", esto es, redenciones luego de una oferta de acciones. Toda vez que, una emisión de acciones muy probablemente mejorará el perfil crediticio del emisor y, consecuentemente, impactará positivamente en la valorización de los bonos HY en el mercado secundario, la práctica del mercado ha sido permitir los equity clawbacks, siempre que estén limitados a un tercio de los bonos en circulación.

En emisiones de bonos grado de inversión ( $y$ en algunas, aunque pocas, emisiones de bonos $\mathrm{HY}$ ) se permite que el emisor redima los bonos en cualquier momento si el emisor paga un precio equivalente a: (i) el valor nominal, más (ii) una prima (conocida como "make-whole premium") equivalente al valor presente de los interés futuros descontados a una tasa igual al rendimiento de los bonos del Tesoro Americano más un spread negociado al momento de emitir los bonos -típicamente menor que el spread utilizado para valorizar los bonos. Esta prima tiene como finalidad compensar a los bonistas por la pérdida esperada producto de la redención anticipada permitiéndoles reinvertir el dinero y recibir un rendimiento similar. Sin embargo, en la práctica, normalmente coloca a los bonistas en una mejor situación porque la tasa de descuento es mucho menor que la tasa de interés de bonos comparables. Por ello, para el emisor, redimir los bonos ejerciendo el make-whole call es sumamente costoso y rara vez será realizado para refinanciar la deuda. Más bien, el makewhole call se ejercerá cuando el emisor quiera librarse de los covenants aplicables a la deuda que quiere redimir.

34. En emisiones de bonos grado de inversión, además del cambio de control, usualmente se requiere una reducción en la clasificación de riesgo por debajo del grado de inversión.

35. The Credit Roundtable, supra nota 22, p. 2. 